\title{
Differences in Oxygen Uptake between Equivalent Resistance Training Protocols: Sets vs. Reps
}

\author{
Christopher B. Scott a, * (D), Maegan Chartier a, Joshua Hodgkiss a, Matthew \\ Mallett ${ }^{a}$, Mikaela Shields a
}

a Exercise, Health and Sport Sciences, University of Southern Maine, Gorham, ME USA

*Corresponding author Email: cscott@maine.edu

DOI: https://doi.org/10.34256/ijpefs20410

Received: 16-12-2020, Revised: 24-12-2020; Accepted: 25-12-2020; Published: 25-12-2020

\begin{abstract}
We examined the energy costs of different resistance training protocols where exercise and recovery periods were equated: 48 total seconds of exercise and 210 seconds of between-set recovery. Two separate investigations were carried out at $65 \%$ of a 1 repetition maximum (1RM): back squat ( 7 men, 3 women) and bench press $(9$ men). Lifting cadence for concentric and eccentric phases was set at $1.5 \mathrm{sec}$ each with $30 \mathrm{sec}$ betweenset recovery periods for the 8 sets, 2 reps protocol (sets) and a $3 \mathrm{~min}$ and $30 \mathrm{sec}$ between-set recovery period for the 2 sets, 8 reps protocol (reps). The amount of oxygen consumed during lifting and between-set recovery periods was significantly greater for sets vs. reps protocol for both the back squat $(+41 \%)$ and bench press $(+27 \%)(p=0.0001)$. Moreover, the total aerobic cost including the after-lifting excess post-exercise oxygen consumption (EPOC) was larger for the increased sets protocol for both the squat $(+27 \%, p=0.01)$ and bench press $(+29 \%, p=0.04)$. Total energy costs - aerobic plus anaerobic, exercise and recovery - were not different among sets or reps protocols. We conclude that a greater volume of oxygen is consumed with a lower repetition, increased number of sets resistance training protocol. We suggest that more recovery periods promote a greater potential for fat oxidation.
\end{abstract}

Keywords: Muscle metabolism, Energy costs, Intermittent exercise, Fat oxidation

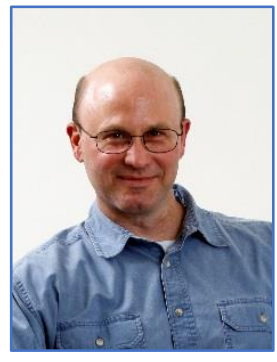

during and after activities.

\section{Introduction}

Exercise programs are designed for a myriad of desired outcomes that may include (but are certainly not limited too) athletic performance, health \& wellness and body composition management. In terms of the latter, both a quantitative and qualitative approach can be made: absolutely in the total amount of calories required of a specific workout and relatively with the preferred use of body fat to fuel the energy requirements of that workout.
High intensity intermittent training (HIIT) appears as a successful strategy in the management of body composition with many HIIT programs consisting of cardiovascular exercise (e.g., running and cycling) $[1,2]$. With its collection of work and rest periods, resistance training certainly falls into the HIIT category. Moreover, regardless of exercise type, it is well known that during the actual exercise period muscle contractile intensity dictates a high anaerobic metabolic contribution consisting of rapid glycolysis (with lactate production) and the usage of the stored high energy phosphates ( $\mathrm{PCr}$ and ATP). Based on such a metabolic response it has been suggested that the successful loss of body fat by HIIT may be directly related not to the actual exercise period, but rather within the recovery periods between sets [3].

With cardiovascular (aerobic) exercise, recovery oxygen consumption immediately and exponentially falls at the end of exercise, demonstrated within exercise science texts as excess post-exercise 
oxygen consumption (EPOC). To the contrary however, with resistance (anaerobic) exercise the greatest rates of oxygen consumption are seen within the first minute of an after-set recovery period when there is typically no muscle contractile intensity $[3,4]$. That is, oxygen uptake actually rises before plummeting after a set of weight lifting.

Based on the different patterns of recovery oxygen uptake between aerobic and anaerobic-type exercises, we asked the question of whether weight lifting programs of equal exercise and rest/recovery time periods, could be designed to modify the amount of over-all oxygen consumed. We hypothesized that if work and rest periods were matched in duration, a lifting protocol with less repetitions and more set-andrecovery periods would result in an increase in the over-all amount of oxygen consumed.

\section{Materials and Methods}

Two equivalent resistance training protocols were created for the back squat and bench press exercises: one using a 2 set, 8 repetition format (reps) and the other an 8 set, 2 repetition (sets) design. Oxygen uptake was measured throughout all exercise and recovery periods. Subjects completed each protocol twice (repeated measurements) with paired analyses that included force $\times$ vertical displacement measurements using a modified Smith machine. Separate projects from two different time periods took place using a convenience sample of 19 participants recruited from a collegiate population. The squat study consisted of 7 males, $21.0 \pm 2.7$ years, $89.0 \pm 17.8 \mathrm{~kg}$, $175.4 \pm 4.8 \mathrm{~cm}$ and 3 females, $23.0 \pm 0.5 \mathrm{yrs}, 60.9 \pm$ $4.6 \mathrm{~kg}, 163.0 \pm 2.1 \mathrm{~cm}$ (Table 1).
For the bench press investigation 9 male subjects volunteered, $23.3 \pm 4.1$ years, $91.6 \pm 16.6$ $\mathrm{kg}, 182.1 \pm 8.9 \mathrm{~cm}$ (Table 2). Each participant had resistance training experience with a frequency of 2-3 times per week for a minimum of 3 months. Both studies were approved by the University of Southern Maine's Institutional Review Board and consent was voluntarily obtained from each participant. For both back squat and bench press exercises, subjects visited the lab on 5 separate occasions.

On the first visit, pre-test instructions were given that included refraining from drinking and eating 4 hours prior to each testing session. On the first visit subjects also practiced a lifting cadence (with no weight) at 1.5 seconds eccentric and 1.5 seconds concentric, as determined by metronome. Subjects then completed a one repetition maximum (1RM) testing procedure on a Smith machine that was used for all data collection. Instructions for 1RM testing were followed using published guidelines [5]. Back squats were performed to a depth where both thighs were parallel with the floor; the bench press movement was completed from the distance of the chest to full arm extension. After 1RM testing, $65 \%$ of the $1 \mathrm{RM}$ was selected as the lifting load for both the back squat and bench press investigations. Anaerobic and aerobic energy cost measurements were completed over the next four laboratory visits. Oxygen uptake measurements were recorded with a metabolic cart (ParvoMedics 2400, Sandy, UT) over the duration of each exercise and between-set recovery protocol and throughout EPOC. The metabolic cart was calibrated for oxygen and carbon dioxide gas percentage and volume before each test. Subjects wore a headpiece attached to a mouthpiece and a nose clip.

Table 1 Subject characteristics: back squat

\begin{tabular}{|c|c|c|c|c|c|}
\hline Gender & Weight $(\mathrm{kg})$ & Height $(\mathrm{cm})$ & Age $(\mathrm{yrs})$ & 1RM $(\mathrm{kg})$ & 1RM \%BW \\
\hline 3 Female & $60.9 \pm 4.6$ & $163.0 \pm 2.1$ & $23.0 \pm 0.5$ & $71.2 \pm 9.4$ & $117 \pm 12$ \\
\hline 7 Male & $89.0 \pm 17.8$ & $175.4 \pm 4.8$ & $21.0 \pm 2.7$ & $127.9 \pm 47.1$ & $144 \pm 61$ \\
\hline 1RM = one repetition maximum; 1RM \%BW = 1RM as a percentage of body weight \\
\hline
\end{tabular}

Table 2 Subject characteristics: bench press

\begin{tabular}{|c|c|c|c|c|c|}
\hline Gender & Weight $(\mathrm{kg})$ & Height $(\mathrm{cm})$ & Age $(\mathrm{yrs})$ & 1RM $(\mathrm{kg})$ & 1RM \%BW \\
\hline 9 Male & $91.6 \pm 16.6$ & $182.1 \pm 8.9$ & $23.3 \pm 4.1$ & $99.6 \pm 31.1$ & $109.1 \pm 27.4$ \\
\hline \multicolumn{7}{|l}{ 1RM = one repetition maximum; 1RM \%BW = 1RM as a percentage of body weight } \\
\hline
\end{tabular}


For the back-squat exercise, expired gas was measured during a 5-minute standing resting period prior to lifting. For the bench press, prior to lifting, a 5minute supine rest period was recorded with subject's feet on the floor and arms placed across the chest. Each subjects resting oxygen uptake was subtracted from all oxygen-consuming exercise, between-set recovery and EPOC components (i.e., net costs).

For the 8 set 2 reps (sets) protocol a 30 second rest period took place between sets; for the 2 set 8 rep (reps) protocol there was a 3.5 minute rest between sets: a total of $48-\mathrm{sec}$ of exercise and $210-\mathrm{sec}$ of between-sets recovery for both squat and bench press protocols. Excess post-exercise oxygen consumption (EPOC) was measured after all sets had been completed.

After the squat exercise, subjects were immediately seated for an EPOC measurement. After the supine bench press was completed, subject's legs were raised to allow a completely supine EPOC period. For both exercises, EPOC measurements continued until oxygen uptake had returned to a standard baseline of $5.0 \mathrm{ml} \mathrm{kg}^{-1} \mathrm{~min}^{-1}$ (a typical standing resting oxygen uptake) or, at the end recovery time period of 15 minutes (whichever came first). Aerobic energy expenditure $(\mathrm{kJ})$ was calculated using the software of the metabolic cart operating system.

Blood lactate measurements were taken with a finger stick at rest and 2 minutes after the completion of each protocol using a hand-held Lactate Scout meter (Leipzig, Germany). Net anaerobic energy costs were determined as the difference between resting and peak blood lactate levels multiplied by $3.0 \mathrm{ml} \mathrm{O}_{2}$ and body weight $(\mathrm{kg})$; this oxygen uptake estimate was then converted as 1 liter $\mathrm{O}_{2}$ uptake $=20.9 \mathrm{~kJ}[6,7]$.

It has been shown that the product of force and vertical displacement best quantifies resistance exercise volume [8]. To that end our Smith machine was modified to measure the distance the bar traveled with a coefficient of variation of $0.25 \%$ between reps and $0.75 \%$ among sets (displacement of less than 1 $\mathrm{mm}$ can be detected). An estimate of Joules ( $\sim$ J) was made that included the product of weight lifted $x$ vertical difference the bar travelled (inertia was not accounted for).

Descriptive analyses and inferential paired ttests were completed between the 8 set, 2 repetition (sets) and 2 set, 8 repetition (reps) protocols; the confidence interval was set at $p=0.05$ or less. For both the back squat and bench press studies, all data set comparisons consisted of the averaged value of two laboratory visits for each protocol (four tests per subject).

\section{Results \& Discussion}

Energy cost comparisons (means \pm standard deviation) and percent differences are shown in table 3 for the back squat exercise. Bench press data (means \pm standard deviation) are shown in table 4 . Net costs are depicted in both tables. Anaerobic costs are based on blood lactate levels. Exercise \& Recovery Aerobic costs are based on oxygen uptake during the exercise and between-set recovery periods. EPOC represents excess post-exercise oxygen consumption after all sets were completed. Total Cost represents all aerobic plus anaerobic cost components. Total Aerobic Costs are the sum of: oxygen-uptake during the lifting periods, between-set recovery periods and EPOC. The product of weight lifted $x$ vertical displacement was used to provide an estimate of Joules ( J). P-values in bold face indicate statistical significance.

We measured the aerobic and anaerobic, exercise and recovery, energy cost components of two separate but equal bouts of submaximal resistance exercise (back squat and bench press at 65\% 1RM) in an attempt to compare the amount of oxygen consumed. With the multiple set protocols, the total amount of oxygen consumed during exercise, between-set recovery and EPOC periods was significantly higher for both the back squat $(27 \%)$ and the bench press (29\%) exercises (Tables 3 and 4).

Our exercise and between-set recovery periods were specifically designed to be equal between set and rep protocols (48-sec of lifting and 210-sec of between-sets recovery). However, the distance the bar traveled between protocols was similar only for the bench press exercise. It appears likely that for the squat (and less so for the bench press) the slight additional distance the weight lifting bar traveled during multiple racking and un-racking of the bar accounted for some of the oxygen-related energy costs. Even so, the $11 \%$ difference in the amount of weight lifted $\times$ vertical displacement $(\sim J)$ between squat protocols fell well below the total aerobic cost difference of $27 \%$, as well as the exercise and between-set recovery oxygen cost difference of $41 \%$; we suggest that the difference in oxygen-related costs between protocols is better related to the multiple-set exercise and recovery (sets) protocol design as opposed to the amount of work performed. 
Table 3 Energy cost components of 2 squat protocols of equal exercise and recovery periods ( 7 male, 3 female subjects)

\begin{tabular}{|c|c|c|c|c|}
\hline & 8 sets 2 reps & 2 sets 8 reps & \% difference & p-value \\
\hline Anaerobic & $\begin{array}{c}11.3 \pm 10.5 \mathrm{~kJ} \\
(3 \pm 3 \mathrm{kcal})\end{array}$ & $\begin{array}{c}19.2 \pm 10.0 \mathrm{~kJ} \\
(5 \pm 2 \mathrm{kcal})\end{array}$ & $70 \%$ & 0.09 \\
\hline $\begin{array}{c}\text { Exer \& Rec } \\
\text { Aerobic }\end{array}$ & $\begin{array}{c}69.0 \pm 22.6 \mathrm{~kJ} \\
(17 \pm 5 \mathrm{kcal})\end{array}$ & $\begin{array}{c}40.2 \pm 11.7 \mathrm{~kJ} \\
(10 \pm 3 \mathrm{kcal})\end{array}$ & $41 \%$ & 0.0001 \\
\hline EPOC & $\begin{array}{c}48.1 \pm 28.5 \mathrm{~kJ} \\
(12 \pm 7 \mathrm{kcal})\end{array}$ & $\begin{array}{c}46.4 \pm 17.2 \mathrm{~kJ} \\
(11 \pm 4 \mathrm{kcal})\end{array}$ & $3 \%$ & 0.82 \\
\hline Total Cost & $\begin{array}{c}128.2 \pm 57.7 \mathrm{~kJ} \\
(31 \pm 14 \mathrm{kcal})\end{array}$ & $\begin{array}{c}106.7 \pm 34.7 \mathrm{~kJ} \\
(26 \pm 8 \mathrm{kcal})\end{array}$ & $17 \%$ & 0.17 \\
\hline $\begin{array}{l}\text { Total Aerobic } \\
\text { Costs }\end{array}$ & $\begin{array}{c}117.0 \pm 47.7 \mathrm{~kJ} \\
(28 \pm 11 \mathrm{kcal})\end{array}$ & $\begin{array}{c}86.6 \pm 25.9 \mathrm{~kJ} \\
(21 \pm 6 \mathrm{kcal})\end{array}$ & $27 \%$ & 0.01 \\
\hline Joules & $639 \pm 365$ & $571 \pm 298$ & $11 \%$ & 0.03 \\
\hline
\end{tabular}

Table 4 Energy cost components of 2 bench press protocols of equal exercise and recovery periods ( 9 male subjects)

\begin{tabular}{|c|c|c|c|c|}
\hline & 8 sets 2 reps & 2 sets 8 reps & $\%$ difference & p-value \\
\hline Anaerobic & $\begin{array}{c}14.1 \pm 7.8 \mathrm{~kJ} \\
(3 \pm 2 \mathrm{kcal})\end{array}$ & $\begin{array}{c}27.6 \pm 9.1 \mathrm{~kJ} \\
(7 \pm 2 \mathrm{kcal})\end{array}$ & $96 \%$ & 0.03 \\
\hline $\begin{array}{c}\text { Exer \& Rec } \\
\text { Aerobic }\end{array}$ & $\begin{array}{c}25.5 \pm 8.6 \mathrm{~kJ} \\
(6 \pm 2 \mathrm{kcal})\end{array}$ & $\begin{array}{c}18.7 \pm 7.3 \mathrm{~kJ} \\
(5 \pm 2 \mathrm{kcal})\end{array}$ & $27 \%$ & 0.0001 \\
\hline EPOC & $\begin{array}{c}31.3 \pm 12.8 \mathrm{~kJ} \\
(8 \pm 3 \mathrm{kcal})\end{array}$ & $\begin{array}{c}21.1 \pm 8.1 \mathrm{~kJ} \\
(5 \pm 2 \mathrm{kcal})\end{array}$ & $36 \%$ & 0.001 \\
\hline Total Cost & $\begin{array}{c}69.3 \pm 17.0 \mathrm{~kJ} \\
(17 \pm 4 \mathrm{kcal})\end{array}$ & $\begin{array}{c}67.2 \pm 17.0 \mathrm{~kJ} \\
(16 \pm 4 \mathrm{kcal})\end{array}$ & $3 \%$ & 0.83 \\
\hline $\begin{array}{l}\text { Total Aerobic } \\
\text { Costs }\end{array}$ & $\begin{array}{c}56.2 \pm 10.5 \mathrm{~kJ} \\
(13 \pm 3 \mathrm{kcal})\end{array}$ & $\begin{array}{c}39.6 \pm 10.8 \mathrm{~kJ} \\
(10 \pm 3 \mathrm{kcal})\end{array}$ & $29 \%$ & 0.04 \\
\hline Joules & $443 \pm 57$ & $435 \pm 45$ & $2 \%$ & 0.79 \\
\hline
\end{tabular}

The greatest relative difference in energy costs between high sets vs. low sets protocols came with the estimation of anaerobic contributions for the bench press, at $96 \%$. This difference may be reflective of a lower metabolic dependence on anaerobic glycolysis for the 8 set, 2 rep bench press protocol: as the number of brief sets increases, the reliance on anaerobic glycolysis with lactate production may decrease, with an equivalent increase in high energy phosphate utilization (PCr and ATP stores) [6].

A large relative difference (70\%) was also found with the anaerobic costs between the two squat protocols, yet perhaps because of the extent of variability involved (this data set included males and females), statistical significance was not found. There is no doubt that blood/muscle lactate levels are at best only an approximation of glycolytic energy expenditure, yet for both back squat and bench press exercises, the total energy costs (aerobic plus anaerobic, exercise and recovery) were not statistically different between equivalent protocols.

In addition to the previously mentioned anaerobic costs, EPOC was statistically different between the two bench press protocols (36\%) but not for the back squat (3\%). During the exercise and between-set recovery periods (excluding EPOC), relative oxygen related costs were different between protocols for both the back squat (41\%) and the bench press $(27 \%)$.

Collectively, we interpret these data as indicating that a multiple set, limited repetition resistance exercise protocol can promote a metabolic scenario where oxygen uptake is increased and glycolysis is steadily diminished, even as overall aerobic plus anaerobic energy costs remain similar. For multiple set protocols, each brief set would be fueled mostly by the stored high energy phosphates, followed with a greater oxygen uptake in the recovery between- 
sets that serves to re-synthesize those PCr-ATP stores [3]. We emphasize that our lifting loads were low, at $65 \%$ of a $1 \mathrm{RM}$; relative aerobic and anaerobic, exercise and recovery energy cost contributions can differ with lifting protocols that result in exhaustion [9].

During the concentric and eccentric phases of resistance exercise, oxygen delivery to working muscle is often compromised, even at relatively low muscular forces so that during the recovery periods between sets, at a time when there is no muscle contraction, oxygen carrying blood flow returns $[10,11]$. These conditions - an increased oxygen supply, a relatively high rate of ATP and $\mathrm{PCr}$ re-synthesis, and little to no muscle contractile force - may create optimal conditions for the oxidation of fat. Additionally, per kilojoule of energy cost, the oxidation of fat $(1 \mathrm{~kJ}=$ 0.050 liters of oxygen) requires slightly more oxygen than does the oxidation of glucose $(1 \mathrm{~kJ}=0.047$ liters of oxygen), so that a greater utilization of fat as a fuel can further serve to elevate the amount of oxygen consumed in recovery [12].

As compared to steady state exercise, the metabolic differences inherent to exercise and recovery created by a high-intensity intermittent exercise (HIIT) protocol helps to explain the success this format has with body composition modification $[1,2,13]$.

\section{Conclusion}

We interpret our data to indicate a greater volume of oxygen being consumed with a high set, low repetition resistance training protocol that necessitates the requirement of numerous recovery periods. This multi-set protocol also appears to reduce glycolytic anaerobic energy contributions over the course of multiple sets while increasing those from the high energy phosphate stores (PCr-ATP) as seen in the form of an increased oxygen uptake during the between-set recovery periods. We suggest that brief intense sets of intermittent exercise followed by obligatory recovery periods promotes further potential for increased fat oxidation.

\section{References}

[1] S.H. Boutcher, High-intensity intermittent exercise and fat loss, Journal of Obesity, 2011; (2011) 868305, DOI | PubMed

[2] F. Maillard, B. Pereira, N. Boisseau, Effect of high-intensity interval training on total, abdominal and visceral fat mass: a metaanalysis. Sports Medicine, 48 (2018) 269-288.

\section{DOI | PubMed}

[3] C.B. Scott, Oxygen costs peak after resistance exercise sets: a rational for the importance of recovery over exercise, Journal of Exercise Physiology, 15 (2012) 1-8.

[4] W.D. McArdle, G.F. Foglia, Energy cost and cardiorespiratory stress of isometric and weight training exercises, Journal of Sports Medicine and Physical Fitness, 9 (1969) 23-30. PubMed

[5] G. Haff, N.T. Triplet, (2015). Essentials of Strength Training and Conditioning (4th ed.). Human Kinetics, Champaign, IL.

[6] C.B. Scott, The effect of time-under-tension and weight lifting cadence on aerobic, anaerobic, and recovery energy expenditures: 3 sets, Applied Physiology, Nutrition, and Metabolism, 37 (2012) 252-256. DOI | PubMed

[7] C.B. Scott, A. Luchini, A. Knausenberger, A. Steitz, Total energy costs - aerobic and anaerobic, exercise and recovery - of five resistance exercises, Central European Journal of Sport Sciences and Medicine, 7 (2014) 53-59.

[8] J.M. McBride, G.O. McCauley, P. Cormie, J.L. Nuzzo, M.J. Cavill, N.T. Triplett, Comparison of methods to quantify volume during resistance exercise, Journal of Strength and Conditioning Research, 23 (2009) 106-110. DOI I PubMed

[9] E.M. Gorostiaga, I. Navarro-Amezqueta, R. Cusso, Y. Hellstern, J.A.L. Calbet, M. Guerrero, C. Granados, M. Gonzalez-Izal, J. Ibanez, M. Izquierdo, Anaerobic energy expenditure and mechanical efficiency during exhaustive leg press exercise, PLoS One 5(10) (2010) e13486. DOI I PubMed

[10] R.H.T. Edwards, D.K. Hill, M. McDonnell, Myothermal and intramuscular pressure measurements during isometric contractions of the human quadriceps muscle, Journal of Physiology, 224 (1972) 58P-59P. PubMed

[11] T. Tetsuro, S. Uchiyama, T. Tamura, S. Nakano, Changes in muscle oxygenation during weightlifting exercise, European Journal of Applied Physiology and Occupational Physiology, 68 (1994) 465-469. DOI I PubMed

[12] C.B. Scott, (2018). How to Maximize the Caloric Costs of Exercise. Archway Publ. Bloomington, IN.

[13] G.R. Hunter, R.L. Weinsier, M.M. Bamman, D.E. Larson, A role for high intensity exercise on energy balance and weight control, Int J Obesity, 22 (1998) 489-493. DOI I PubMed 


\section{Acknowledgement}

We thank Filip Manjencic for help with data collection (back squat study) along with Matthew Gerry and Ben Garelick (bench press study).

\section{Funding}

Nil

\section{Authors Contribution}

Individual contributions are as follows: Christopher B. Scott - Study Design, Manuscript Preparation, Review \& Editing. Data Collection; M Maegan Chartier, Joshua Hodgkiss, Matthew Mallett and Mikaela Shields. All authors have read and approved the manuscript.

\section{Ethics Approval}

This study was approved by Institutional Ethics Committee

\section{Conflict of interest}

Nil

Availability of data and material

No additional data are available

Informed consent

Written consent was obtained from the participants

\section{About The License}

(C) The author(s) 2020. The text of this article is open access and licensed under a Creative Commons Attribution 4.0 International License 